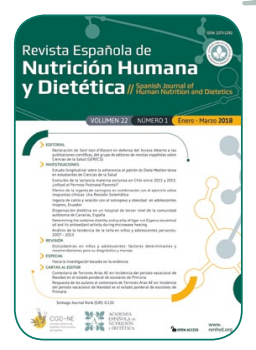

\title{
CARTA AL EDITOR \\ Comentario de Terrones Arias AE en: Incidencia del periodo vacacional de Navidad en el estado ponderal de escolares de Primaria
}

\author{
Angel Eduardo Terrones Arias ${ }^{\mathrm{a}}$, Oliverio Carbajal Huamani ${ }^{\mathrm{a}, *}$ \\ aniversidad Peruana de Ciencias Aplicadas, Los Álamos de Monterrico, Santiago de Surco, Lima. \\ *oliverio_crb@hotmail.com
}

Editor Asignado: Rodrigo Martínez-Rodríguez. Universidad de Murcia. Murcia, España.

Recibido el 25 de noviembre de 2017; aceptado el 16 de marzo de 2018; publicado el 18 de marzo de 2018.

\section{CITA}

Terrones Arias AE, Carbajal Huamani O. Comentario de Terrones Arias AE en: Incidencia del periodo vacacional de Navidad en el estado ponderal de escolares de Primaria. Rev Esp Nutr Hum Diet. 2018; 22(1): 101-2. doi: 10.14306/ renhyd.22.1.521

Sr. Editor: hemos leído con interés el artículo titulado "Incidencia del periodo vacacional de Navidad en el estado ponderal de escolares de Primaria"1 y consideramos que el título puede llevar a confusión. Esto debido a que Incidencia significa presencia de nuevos $\operatorname{casos}^{2}$ y en este trabajo se usa/menciona el término "Incidencia del periodo vacacional" y consideramos que no es adecuado, porque lo que se evalúa es la variación del estado ponderal (peso, talla e IMC).

Por otro lado, para evaluar incidencia (nuevos casos), se debería haber evaluado nuevos casos de sobrepeso u obesidad. Sin embargo, esto no se ha presentado y entendemos que es debido a la corta duración de la observación (40 días en total, 20 días después del periodo vacacional de Navidad y 20 días después de regresar a clases). En este tiempo se espera que el cambio de categoría del estado nutricional no sea significativo, salvo que se encuentren en el límite del punto de corte que los lleve a pasar a la otra clasificación del estado ponderal ${ }^{3}$.

De igual manera, consideramos que en la tabla 1 es irrelevante mostrar los valores $p$ que corresponden al " $p$-valor (tiempo x grupo)" en las variables de peso e IMC, por nivel de IMC (G1: Normopeso, G2: Sobrepeso; G3: Obesidad) pues de por sí son los esperados. Por otro lado, el valor p que corresponde a "p-valor (grupo x tiempo)" en la variable de talla, también creemos que no es relevante, puesto que el cambio $1 \mathrm{~cm}$ entre mediciones puede darse por error de medición. 
Por otra parte, llama la atención que dada la escasa población en los G2 (18 alumnos) y G3 (19 alumnos) el resultado de la prueba ANOVA indique un "valor $p$ " muy bajo, ante diferencias muy pequeñas. Por ejemplo: la variable talla, muestran diferencias significativas (según subíndices) entre tiempo T2 y T3 en los grupos G1 y G3 cuando los valores de talla y su desviación estándar son iguales; y en el caso de la variable IMC en el G1 entre T2 y T3, una diferencia de sólo 0,07 de IMC muestra un $p$ de 0,002.

Por último, si se aclaran los puntos expuestos en observación creemos que el trabajo aportaría de mejor manera a futuras investigaciones.

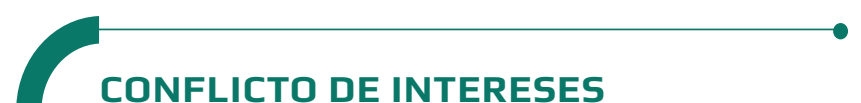

Los autores expresan que no existen conflictos de interés al redactar el manuscrito.

\section{REFERENCIAS}

(1) Martínez-Redondo M, Latorre-Román PÁ. Incidencia del periodo vacacional de Navidad en el estado ponderal de escolares de Primaria. Rev Esp Nutr Hum Diet. 2017; 21(3): 230-6.

(2) Hernández-Aguado I, Gil A, Delgado M, Bolúmar F, Benavides $\mathrm{F}$, Porta $\mathrm{M}$, et al. Manual de epidemiología y salud pública: para grados en ciencias de la salud. 2a ed. Madrid: Médica Panamericana; 2011.

(3) Zurita JN, Díaz I, Nishimura E, Villasis MÁ, Rivera AJ, Garrido E. Change in body mass index among girls with precocious puberty under treatment. Arch Argent Pediatr. 2016; 114(2): 143-5. 